\title{
Ab initio calculations of F-H-Br system with linear geometry
}

\author{
Dmytro Babyuk $^{\mathrm{a}^{*}}$, Jacek Korchowiec ${ }^{\mathrm{b}}$ and Yaryna Motovylina ${ }^{\mathrm{a}}$
}

${ }^{a}$ Chernivtsi national university, 2 Kotsyubinski Str., 58012 Ukraine

${ }^{b}$ Jagiellonian university in Cracow, 3 Ingardena Str., Cracow, Poland

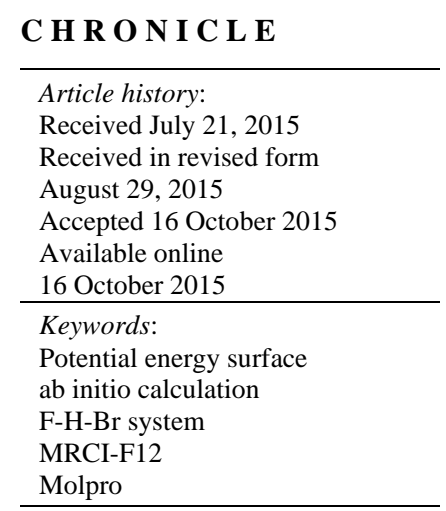

\begin{abstract}
A B S T R A C T
Two potential energy surfaces $1^{2} \mathrm{~A}_{1}$ and $1^{2} \mathrm{~B}_{1}$ for linear geometry of $\mathrm{F}-\mathrm{H}-\mathrm{Br}$ system have been computed with aug-cc-pVQZ basis set using dynamically weighted state averaged MCSCF followed by MRCI-F12 method. State $1{ }^{2} \mathrm{~A}_{1}$ has smaller barrier height $(3.49 \mathrm{kcal} / \mathrm{mol})$ than $1{ }^{2} \mathrm{~B}_{1}$. (13.6 kcal/mol). The latter has deep van der Waals well in Br-HF valley $(2.12 \mathrm{kcal} / \mathrm{mol})$.
\end{abstract}

\section{Introduction}

Reactions between a halogen atom and hydrogen halide diatomic molecule present a special interest. Many chain reactions comprise them as propagation step. However, first of all an accurate global potential energy surface (PES) is required to initiate the computation. There has been a number of PESs for systems F-H-F ${ }^{1-2}, \mathrm{~F}-\mathrm{H}-\mathrm{Cl}^{3}$, Cl-H-Cl${ }^{4-7}, \mathrm{Br}-\mathrm{H}-\mathrm{Br}^{8}$. Due to open-shell character of such systems multiple electronic states are needed to properly describe the dynamics. To our knowledge systems with bromine and/or iodine atoms were studied using only semiempirical PESs ${ }^{9-10}$. Therefore the goal of this paper is to obtain the accurate $a b$ initio PESs for F-H-Br system. Despite the presence of heavy atom, we neglect here by all relativistic effects. Also in this work only linear arrangement of all atoms is considered. Thus this paper represents a first step toward global $a b$ initio surfaces for F-H$\mathrm{Br}$ system. The chosen arrangement can be used in the description of the following collinear reactions

$$
\begin{aligned}
& \mathrm{F}+\mathrm{HBr} \rightarrow \mathrm{HF}+\mathrm{Br} \\
& \mathrm{Br}+\mathrm{HF} \rightarrow \mathrm{HBr}+\mathrm{F}
\end{aligned}
$$


Within chosen linear geometry the molecular system belongs to the $\mathrm{C}_{2 \mathrm{v}}$ point group symmetry. Three lower electronic states are ${ }^{2} \Sigma\left(1{ }^{2} \mathrm{~A}_{1}\right),{ }^{2} \Pi_{x}\left(1^{2} \mathrm{~B}_{1}\right)$ and ${ }^{2} \Pi_{y}\left(1^{2} \mathrm{~B}_{2}\right)$ asymptotically correlate to the ground states of $\mathrm{F}\left({ }^{2} \mathrm{P}\right)+\mathrm{HBr}$ and $\operatorname{Br}\left({ }^{2} \mathrm{P}\right)+\mathrm{HF}$. The degenerate states ${ }^{2} \Pi_{x}$ and ${ }^{2} \Pi_{y}$ are lower at long ranges but in the vicinity of the transition state (TS) area the energy ordering becomes $E\left({ }^{2} \Sigma\right)<E\left({ }^{2} \Pi_{x}\right)$. Since these states belong to different symmetry species they cross at some intermediate distances. Such energy reordering is due to different electrostatic interaction. In the TS region the singly occupied porbital of the $\mathrm{F}$ atom pointing directly towards the $\mathrm{H}$ atom of $\mathrm{HBr}\left({ }^{2} \Sigma\right.$ state) gives rise to a more attractive potential than perpendicular singly occupied p-orbital $\left({ }^{2} \Pi_{x}\right.$ state) to the FHBr axis. The latter orientation leads to stronger electrostatic interactions at long range. Deviation from linearity reduces the symmetry to $C_{s}$ and now both ${ }^{2} \Sigma$ and ${ }^{2} \Pi_{x}$ become the same symmetry states $1{ }^{2} \mathrm{~A}^{\prime}$ and $2{ }^{2} \mathrm{~A}^{\prime}$, respectively. They cannot cross anymore and form avoided crossing region.

\section{Computational Methods}

The geometric configuration of the studied molecular system and notations for internal coordinates is presented in Figure 1. Only two coordinates are needed because as mentioned above it is implied that all three atoms lie along a straight line. These coordinates are interatomic distances $R_{1}$ and $R_{2}$ as shown in Fig. 1.

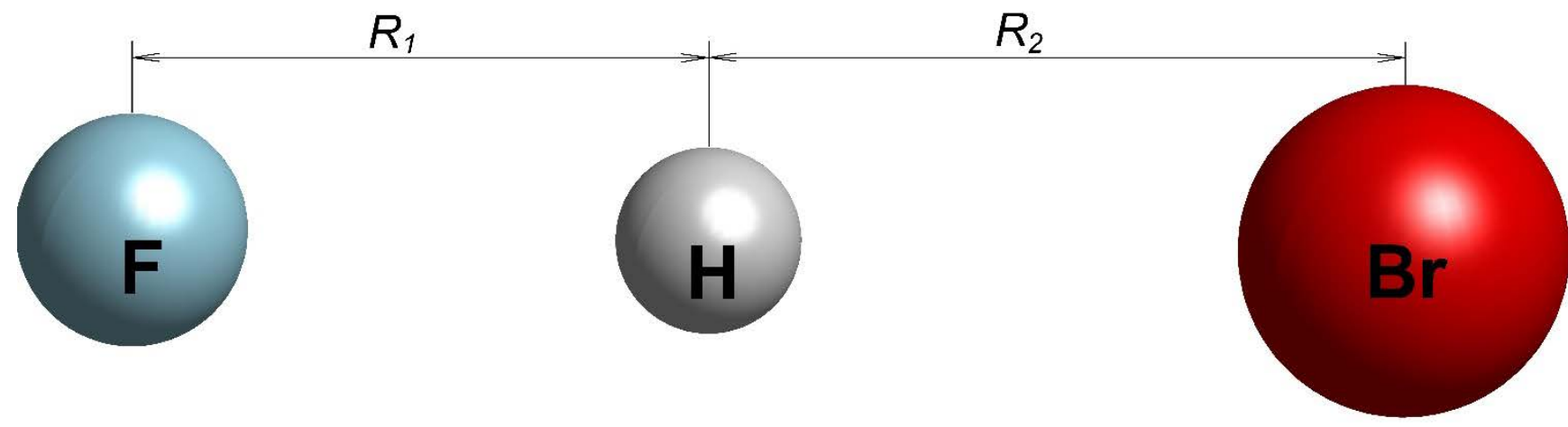

Fig. 1. Geometry of the studied molecular system

All calculations were carried out using Molpro2012 package $^{11}$. Initial states were calculated using state-averaged multi-configurational self-consistent field (SA-MCSCF) with aug-cc-pVTZ and aug-ccpVQZ basis sets. As was shown for similar system $\mathrm{F}-\mathrm{H}-\mathrm{Cl}^{3}$, in the TS area states with charge transfer also must be included in state averaging in order to fully describe molecular system. Therefore a total of six states are included in the dynamically weighting SA-MCSCF calculation (two lowest of each of the following: ${ }^{2} \mathrm{~A}_{1},{ }^{2} \mathrm{~B}_{1},{ }^{2} \mathrm{~B}_{2}$ ) with the decay coefficient $\beta^{-1}=2.78 \mathrm{eV}^{12}$. The active space includes 8 active orbitals $\left(4 A_{1}, 2 B_{1}, 2 B_{2}, 0 A_{2}\right)$. One valence orbital of $A_{1}$ symmetry is closed to excitation in order to keep consistent ordering of orbitals in Molpro and avoid discontinuities in the PESs ${ }^{3}$. Figure 2 shows six SA-MCSCF states at fixed value of $R_{2}=3.30 a_{0}$. As seen, states $1^{2} \mathrm{~A}_{1}$ and $1{ }^{2} \mathrm{~B}_{1}$ cross at some point. However, the same symmetry states $1^{2} \mathrm{~B}_{1}$ and $2{ }^{2} \mathrm{~B}_{1}$ experience avoided crossing. These conical interaction seams make these surfaces unsmooth.

Since MCSCF does not provide sufficiently accurate results, further computations are required. Usually multi-reference configuration interaction (MRCI) ${ }^{13}$ is then employed with initial states obtained within the MCSCF calculation. Our focus is restricted only on three lowest states $1{ }^{2} \mathrm{~A}_{1}, 1^{2} \mathrm{~B}_{1}$ and $1{ }^{2} \mathrm{~B}_{2}$ (actually two states, because $1{ }^{2} \mathrm{~B}_{1}$ and $1{ }^{2} \mathrm{~B}_{2}$ are degenerate everywhere). Instead of conventional MRCI method we used explicitly correlated MRCI-F12 ${ }^{14}$. This method employs wave functions that explicitly depend on the electron-electron distance. As a result, it has better convergence with basis set. For example, the MRCI-F12 method yields results with near complete basis set limit 
accuracy already with triple- $\zeta$ basis sets. So the obtained dynamically weighted SA-MCSCF orbitals were used as references in MRCI-F12. The Davidson correction $(+Q)$ was accounted as well.

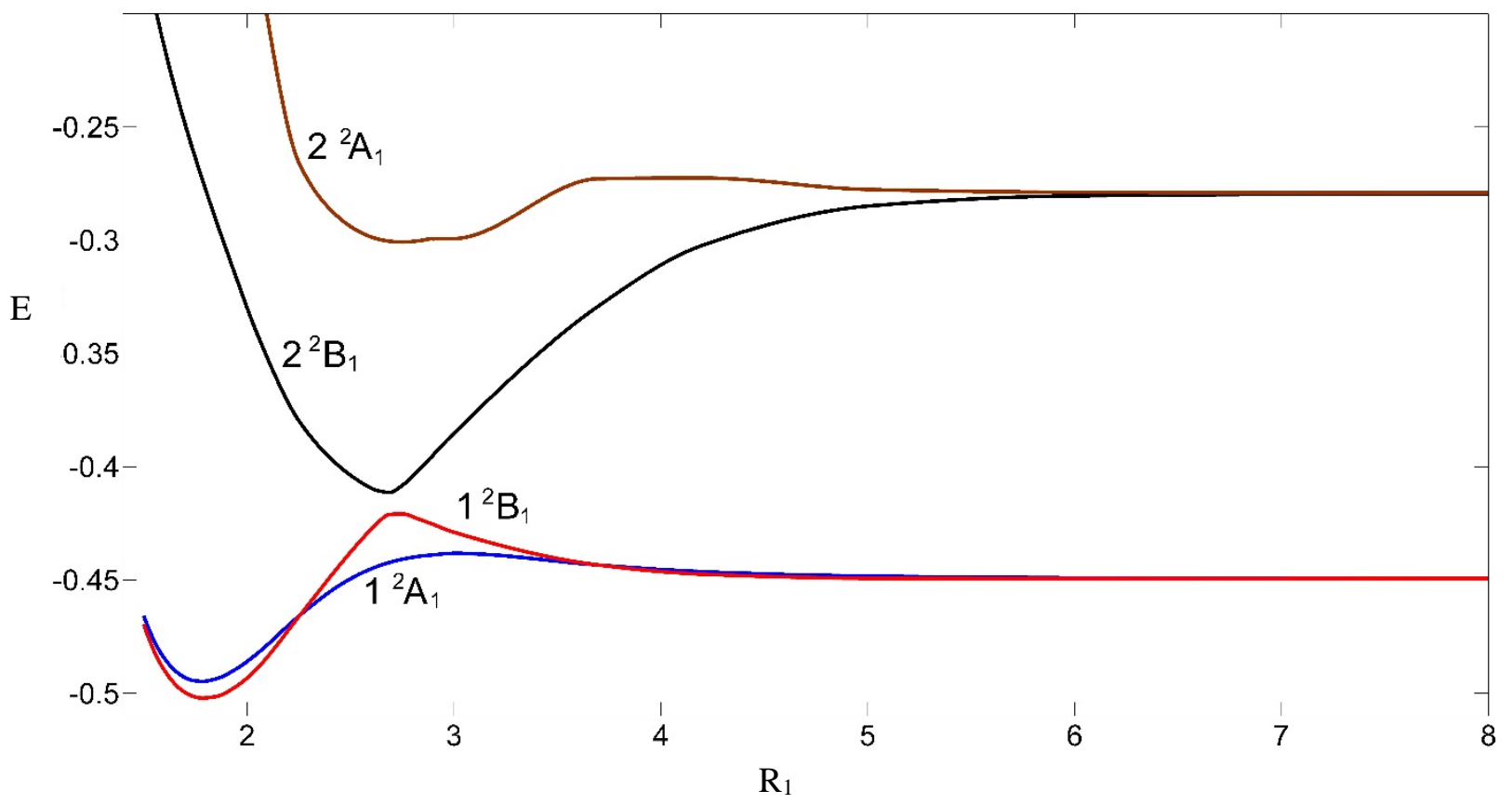

Fig. 2. Six SA-MCSCF states $\left(1^{2} \mathrm{~B}_{2}\right.$ and $2{ }^{2} \mathrm{~B}_{2}$ coincide with $1{ }^{2} \mathrm{~B}_{1}$ and $2{ }^{2} \mathrm{~B}_{1}$, respectively $)$ at $R_{2}=3.30 a_{0}$ using aug-cc-pVQZ basis set.

A rectangular grid of points $R_{1}=[1.35-14.0] a_{0}$ and $R_{2}=[2.0-14.0] a_{0}$ was constructed with total number of 567 nodes. The density of the grid was larger in the TS and asymptotic regions. It is necessary to properly sample these important areas of the PESs. Then 2D-cubic spline interpolation was performed using MATLAB® suite and two PESs were generated.

\section{Results and Discussion}

The first test for $a b$ initio calculations is comparison of the asymptotic parameters of the PESs with experimental data. Of course, both PESs coincide here.

Table. 1. Parameters for the diatomic molecules derived from the PESs at asymptotic regions compared with experimental data ( $D_{e}$ - dissociation energy, $R_{e}$ - equilibrium bond length, ZPE zero point energy, $\omega_{e}$ - harmonic frequency).

\begin{tabular}{|c|c|c|c|c|c|}
\hline & Basis set & $D_{e}, \mathrm{kcal} / \mathrm{mol}$ & $R_{e}, a_{0}$ & ZPE, kcal/mol & $\omega_{e}, \mathrm{~cm}^{-1}$ \\
\hline & aug-cc-pVTZ & 139.07 & 1.744 & 5.93 & 4144.7 \\
\hline \multirow[t]{3}{*}{$\mathrm{HF}$} & aug-cc-pVQZ & 139.24 & 1.732 & 5.92 & 4138.2 \\
\hline & Experiment $^{3}$ & 141.63 & 1.733 & 5.96 & 4138.3 \\
\hline & aug-cc-pVTZ & 92.77 & 2.696 & 3.83 & 2677.3 \\
\hline \multirow[t]{2}{*}{$\mathrm{HBr}$} & aug-cc-pVQZ & 92.94 & 2.692 & 3.85 & 2690.5 \\
\hline & Experiment $^{15}$ & 93.88 & 2.673 & - & 2649.3 \\
\hline
\end{tabular}


Table 1 summarizes the dissociation energies, equilibrium distances, zero point energies and harmonic frequencies for two diatomic molecules $\mathrm{HF}$ and $\mathrm{HBr}$. The experimental dissociation energy for $\mathrm{HBr}$ is given without spin-orbit effect because our calculations neglect it. As seen, there are no significant discrepancies between the results for both basis sets and it would be sufficient to run all computations only with aug-cc-pVTZ basis. However, all further computations used aug-cc-pVQZ basis. It is important to note that even with such a large basis set the computed dissociation energies are not in excellent agreement with the experimental ones. This is not a limitation of MRCI-F12 or basis set convergence but rather the size of the active space. In order to achieve the accuracies of 0.1 $\mathrm{kcal} / \mathrm{mol}, 3 \mathrm{sF}$ and $3 \mathrm{pF}$ orbitals are to be included into the active space. But this means that all $4 \mathrm{dBr}$ orbitals also must be included thus significantly increasing the number of occupied orbitals and making the computation prohibitively expensive. Another check is comparison of exothermicity for $\mathrm{F}+\mathrm{HBr} \rightarrow \mathrm{Br}+\mathrm{HF}$ reaction. According to Table 2 the computed $\Delta H$ agrees well with the experimental value.

Table. 2. Transition state barrier height ( $\left.E_{\text {barr }}\right)$, energy difference $(\Delta E)$, and exothermicity $(\Delta H)$ of reaction (1)

\begin{tabular}{cccc}
\hline Basis set & $E$ barr, $\mathrm{kcal} / \mathrm{mol}$ & $\Delta E, \mathrm{kcal} / \mathrm{mol}$ & $\Delta H, \mathrm{kcal} / \mathrm{mol}$ \\
\hline aug-cc-pVTZ & 3.39 & -46.30 & -48.40 \\
aug-cc-pVQZ & 3.49 & -46.31 & -48.38 \\
Experiment $^{9}$ & - & - & -48.50 \\
\hline
\end{tabular}

Fig. 3a shows the contour plots of $1{ }^{2} \mathrm{~A}_{1}$ PES. It is smooth and does not have van der Waals (vdW) wells and conical seams. The TS is located at $R_{1}=2.85 a_{0}, R_{2}=2.74 a_{0}$ and is of $3.49 \mathrm{kcal} / \mathrm{mol}$ high. The $1{ }^{2} \mathrm{~B}_{1}$ PES has qualitative differences (Figure $3 \mathrm{~b}$ ). It has the vdW wells in both reactants and product valleys. Also it has conical intersection seams. The saddle point is shifted towards lower F-H and higher $\mathrm{H}$-Br distances $\left(R_{1}=2.58 a_{0}, R_{2}=2.86 a_{0}\right)$ and the barrier is much higher namely $13.6 \mathrm{kcal} / \mathrm{mol}$.
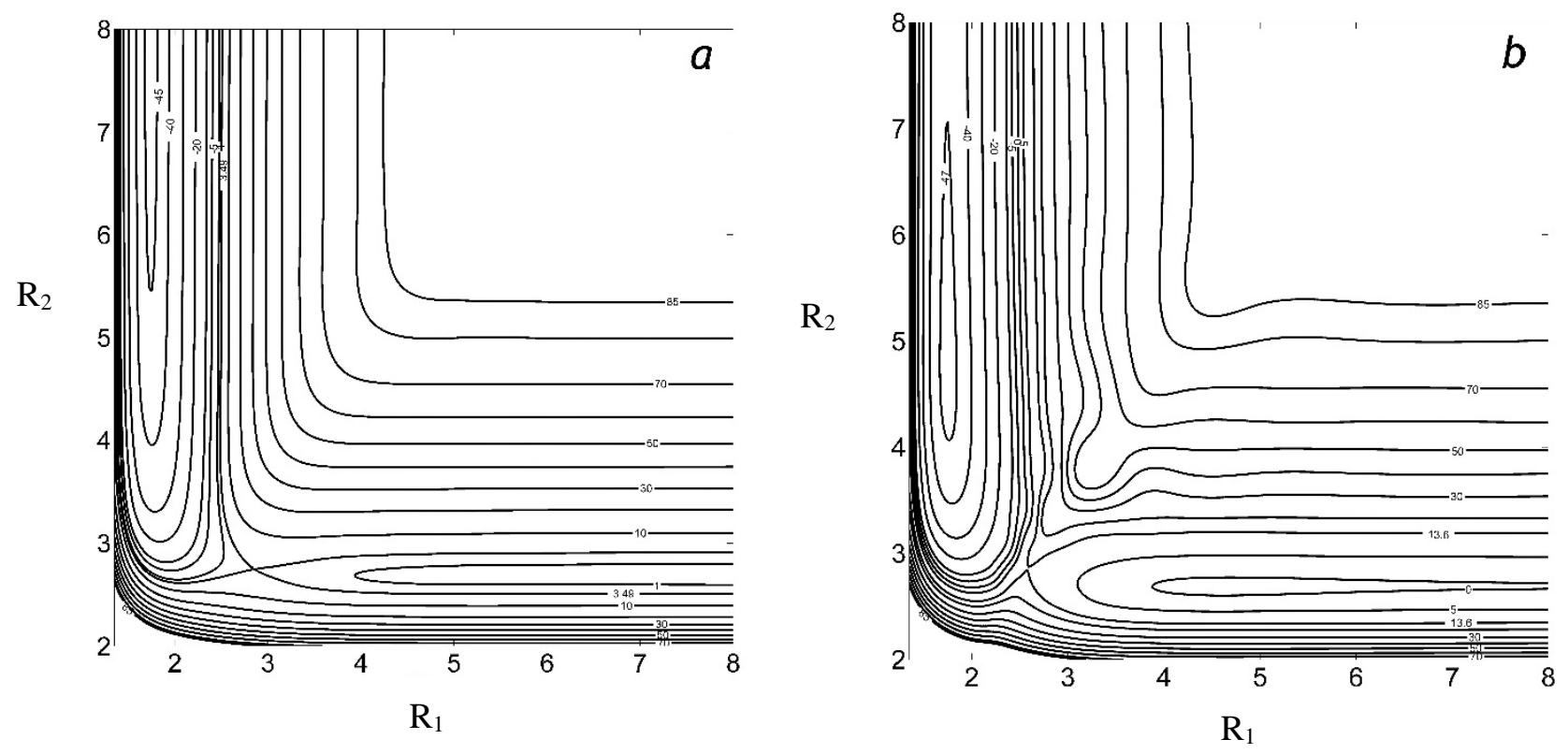

Fig. 3. Contours of $1^{2} \mathrm{~A}_{1}(a)$ and $1^{2} \mathrm{~B}^{1}(b)$ PESs. The numbers at contour lines represent energy in $\mathrm{kcal} / \mathrm{mol}$

The avoided crossing makes this surface discontinuous. Unfortunately these discontinuities lie close to the TS area. But since the barrier height for $1{ }^{2} \mathrm{~A}_{1}$ PES is lower, it would not affect the reaction dynamics for this linear configuration. 
The $1{ }^{2} \mathrm{~A}_{1}$ PES does not exhibit any vdW wells in both reactant and product valleys. It is due to absence of dipole- and quadrupole-quadrupole interaction for this symmetry. However, the $1{ }^{2} \mathrm{~B}_{1}$ state has this sort of interaction leading to two vdW wells. The reactant region exhibits a F-HBr well depth of $0.62 \mathrm{kcal} / \mathrm{mol}$ at $R_{1}=4.66 a_{0}, R_{2}=2.69 a_{0}$ (Fig. 4).

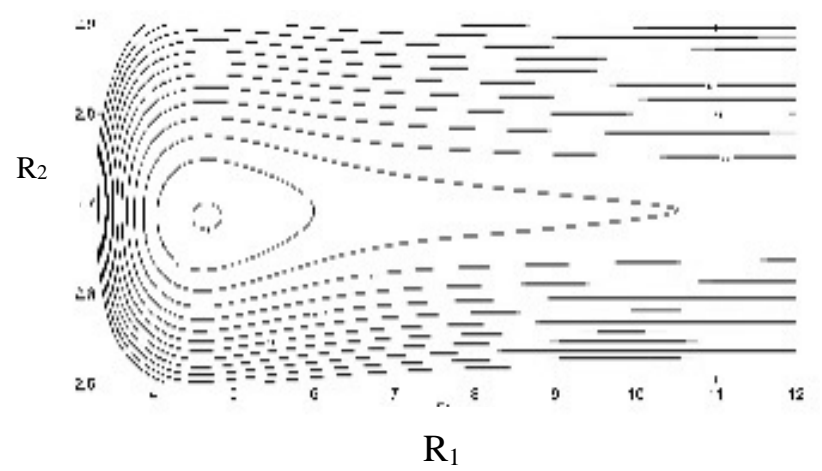

Fig. 4. The vdW well in the reactant valley of $1{ }^{2} \mathrm{~B}_{1}$ PES

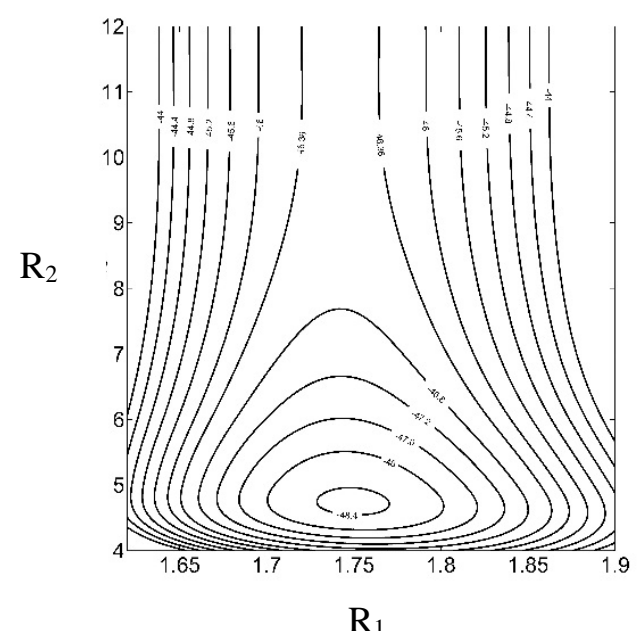

Fig. 5. The vdW well in the product valley of $1{ }^{2} \mathrm{~B}_{1}$ PES

The product valley has much deeper well of $2.12 \mathrm{kcal} / \mathrm{mol}$ with respect to the asymptote. It is located at $R_{1}=1.75 a_{0}, R_{2}=4.66 a_{0}$ (Fig. 5). Similar results are derived for the F-H-Cl system ${ }^{3}$. The wells are 0.67 and $1.95 \mathrm{kcal} / \mathrm{mol}$ deep for reactant and product valleys, respectively. Stronger vdW well for Br-HF complex is due to larger bromine atom size. The presence of such vdW wells significantly influences reaction dynamics ${ }^{16}$.

\section{Conclusions}

This paper presents results of $a b$ initio computation for linear geometry of F-H-Br system. Two lower states $1^{2} \mathrm{~A}_{1}$ and $1{ }^{2} \mathrm{~B}_{1}$ (which are degenerate in asymptotic regions) were computed using dynamical weighted SA-MCSCF method followed by MRCI-F12 calculation. Even though this method provides better convergence with basis set than conventional MRCI, the correlation energy is not fully extracted. Better agreement with experimental data is possible if larger portion of the correlation energy is extracted. This can be accomplished only by employment of larger active space. However, in this case the computations may become prohibitively expensive.

State $1{ }^{2} \mathrm{~A}_{1}$ has much lower barrier height than state $1{ }^{2} \mathrm{~B}_{1}$. As a result, the reaction dynamics in the TS region will be mainly governed by $1{ }^{2} \mathrm{~A}_{1}$ state. However, at long distances between the atom and diatom the dynamics is influenced by both states. A special effect comes from $1^{2} \mathrm{~B}_{1}$ state due to the vdW well. As shown in work ${ }^{16}$, its presence can quantitatively change the system behavior. Therefore the full reactive dynamics for this system implies non-adiabatic study on two coupled PESs. The number of these PESs grows with deflection from linear configuration. In this case the symmetry reduces and states $2{ }^{2} \mathrm{~A}^{\prime}$ and $1^{2} \mathrm{~A}^{\prime \prime}\left({ }^{2} \mathrm{~B}_{1}\right.$ and $1{ }^{2} \mathrm{~B}_{2}$ for linear arrangement, respectively) are not degenerate anymore. Moreover, since now states $1^{2} \mathrm{~A}^{\prime}$ and $2{ }^{2} \mathrm{~A}^{\prime}$ belong to the same symmetry, they form avoided crossings. Ab initio calculations for global PESs with subsequent study of the reactive dynamics are planned for our future research. 


\section{Acknowledgments}

The authors are deeply grateful to PLGrid infrastructure (grants plgdbabyuk2014a and plgdbabyuk2014b) for providing access to computing resources.

\section{References}

1. Tanaka T., Takayanagi T. (2010) Quantum reactive scattering calculations of $\mathrm{H}+\mathrm{F}_{2}$ and $\mathrm{Mu}+\mathrm{F}_{2}$ reactions on a new ab initio potential energy surface. Chem. Phys. Lett., 496 (4-6) 248-253.

2. Bittererová M., Biskupič S., Lischka H., Jakubetz W. (2000) The barrier topography of the H + F2 potential energy surface. Phys. Chem. Chem. Phys., 2 (4) 513-521.

3. Deskevich M. P., Hayes M. Y., Takahashi K., Skodje R. T., and Nesbitt D.J. (2006) Multireference configuration interaction calculations for the $\mathrm{F}\left({ }^{2} \mathrm{P}\right)+\mathrm{HCl} \rightarrow \mathrm{HF}+\mathrm{Cl}\left({ }^{2} P\right)$ reaction: A correlation scaled ground state $\left(1^{2} A^{\prime}\right)$ potential energy surface. J. Chem. Phys., 124 (22) 224303.

4. Maierle C. S., Schatz G. S., Gordon M. S., McCabec P., and Connor J. N. L. (1997) Coupled potential-energy surfaces and quantum reactive scattering for the $\mathrm{Cl}\left({ }^{2} \mathrm{P}\right)+\mathrm{HCl} \rightarrow \mathrm{ClH}+\mathrm{Cl}\left({ }^{2} \mathrm{P}\right)$ reaction. J. Chem. Soc. Faraday Trans., 93 (5) 709-720.

5. Schatz G.S., Hankel M., Whiteley T. W. J., and Connor J. N. L. (2003) Influence of spin-orbit effects on chemical reactions: Quantum scattering studies for the $\mathrm{Cl}\left({ }^{2} \mathrm{P}\right)+\mathrm{HCl} \rightarrow \mathrm{ClH}+\mathrm{Cl}\left({ }^{2} \mathrm{P}\right)$ reaction using coupled ab initio potential energy surfaces. J. Phys. Chem. A, 107 (37) 7278-7289.

6. Whiteley T. W. J., Dobbyn A.J., Connor J. N. L., and Schatz G. C. (2000) Quantum scattering on coupled ab initio potential energy surfaces for the $\mathrm{Cl}\left({ }^{2} \mathrm{P}\right)+\mathrm{HCl} \rightarrow \mathrm{ClH}+\mathrm{Cl}\left({ }^{2} \mathrm{P}\right)$ reaction. Phys. Chem. Chem. Phys., 2 (4) 549-556.

7. Dobbyn A.J., Connor J. N. L., Besley N.A., Knowles P. J., and Schatz G. C. (1999) Coupled ab initio potential energy surfaces for the reaction $\mathrm{Cl}\left({ }^{2} \mathrm{P}\right)+\mathrm{HCl} \rightarrow \mathrm{ClH}+\mathrm{Cl}\left({ }^{2} \mathrm{P}\right)$. Phys. Chem. Chem. Phys., 1 (6) 957-966.

8. Takayanagi T. (2007) Theoretical study of the $\mathrm{H}+\mathrm{Br} 2$ and $\mathrm{Mu}+\mathrm{Br} 2$ reactions: A new ab initio potential energy surface and quantum dynamics calculations. Chem. Phys., 334 (1-3) 109-116.

9. Kornweitz H, and Persky A (2004) Quasiclassical Trajectory Calculations for the Reactions F + $\mathrm{HCl}, \mathrm{F}+\mathrm{HBr}$, and F + HI. J. Phys. Chem. A, 108 (1) 140-145.

10. Duan Z.X., Qi Y., and Lu Y.C. (2008) Theoretical study of the stereodynamics for the reaction F+HBr. Mol. Phys. 106 (24) 2725-2731.

11. Werner H.-J., Knowles P. J., Knizia G., Manby F. R., Schütz M., and others MOLPRO, version 2012.1, a package of ab initio programs.

12. Deskeich M. P., Nesbitt D. J., Werner H.-J. (2004) Dynamically weighted multiconfiguration selfconsistent field: Multistate calculations for $\mathrm{F}+\mathrm{H}_{2} \mathrm{O} \rightarrow \mathrm{HF}+\mathrm{OH}$ reaction paths. J. Chem. Phys., 120 (16) 7281-7289.

13. Werner H.-J., and Knowles P.J. (1988) An efficient internally contracted multiconfigurationreference configuration interaction method. J. Chem. Phys., 89 (9) 5803-5812.

14. Shiozaki T., Knizia G., Werner H.-J. (2011) Explicitly correlated multireference configuration interaction: MRCI-F12. J. Chem. Phys., 134 (3) 034113.

15. de Oliveira-Filho A. G. S., Ornellas F. R., and Peterson K. A. (2012) Accurate ab initio potential energy surfaces for the $3 \mathrm{~A}^{\prime \prime}$ and $3 \mathrm{~A}^{\prime}$ electronic states of the $\mathrm{O}\left({ }^{3} \mathrm{P}\right)+\mathrm{HBr}$ system. J. Chem Phys. 136 (17) 174316.

16. Babyuk D.P., Nechiporuk V. V. (2012) Study of the total reactive dynamics of the H + DCl reaction within the framework of the quantum approach. Russian J. Phys. Chem. B, 6 (1) 1-4. 